\title{
Off-label use of approved drugs
}

A few months ago I received an e-mail from Frank Meyskens, a former trainee of mine. He and his colleagues had recently published a dynamite paper on a randomized, placebo-controlled trial (Meyskens, F. L. Jr et al. Cancer Prev. Res. 1, 32-38 [2008]), which showed that a combination of difluoromethylornithine and sulindac-two drugs that have been around and in the clinic for 30 years-could reduce the recurrence of adenomas in people who had colonoscopic removal of adenomas. The reduction in recurrences was huge and highly significant. There was no difference in adverse effects between the treatment and placebo arms after 3 years. This is a first for "combination chemoprevention", a concept proposed by Mike Sporn in the 1980s.

Meyskens is preparing to take the regulatory steps necessary to get this drug combination approved for use in the clinic, and he wanted my advice on how to do this. What would the FDA ask for? How did you get MOPP (mechlorethamine, vincristine, procarbazine, prednisone), the first effective combination chemotherapy for Hodgkin disease, approved, he asked? The answer is, we never asked the FDA for advice on what was required to cure Hodgkin disease, and MOPP was never submitted to the FDA for approval. Three of the four drugs in the MOPP regimen were old, well known and available, and the fourth was already known to be effective from results of phase II studies in Hodgkin disease. The FDA approved it later. How we used the MOPP regimen was different. MOPP was an example of off-label use of drugs in an investigative protocol by experienced clinical investigators. It worked, was quickly adopted by practicing physicians, and led to a dramatic $70 \%$ reduction in mortality from Hodgkin disease in less than a decade. Had we followed current procedure, we would most assuredly have had to modify what we thought was a good design to satisfy inexperienced committees everywhere, and would have had to test each component of MOPP against the MOPP regimen. We would surely have lost a decade, and many people alive today would be dead.

I had forgotten about Frank's query until I saw a frontpage article in the New York Times on 27 January 2009 entitled "Medicare widens drugs it accepts for cancer care." It pointed out that Medicare was now going to pay for more off-label use of some cancer drugs, uses that have not been approved by the FDA. Good for them! Critics, the article pointed out, were upset with this because these drugs would now be used with little evidence of benefit. That is not strictly true. Even unapproved drugs often show early evidence of benefit reliable enough to use in desperately ill cancer patients. However, this evidence is not of the type that a company needs for a new drug application. 'No evidence' often means not studied enough, or too little profit to go through the bother. Off-label testing of approved drugs in combination should not be an FDA regulated exercise in the first place.

It would be like asking the FDA to approve every change in the schedule for the use of radiotherapy. We got to this point by assuming we had to ask for all these things. Until we go back to the 'good old days', investigators should not be trying to figure out what the FDA might require. They should do what they think is right and necessary. The difluoromethylornithine and sulindac combination used by Meyskens and colleagues should require approval only by their institution's Human Investigation Committee. People at risk for colon cancer should not have to wait a decade to use drugs that have been around for three decades. That is the advice I gave to Frank Meyskens.

doi:10.1038/nrclinonc.2009.41
VT DeVita Jr is the Editor-in-Chief of Nature Reviews Clinical Oncology.

Competing interests The author declared no competing interests. 